\title{
Lorentz contracted proton
}

\section{Bedoya Fierro, N.G. Kelkar and M. Nowakowski}

Dept. de Fisica, Universidad de los Andes, Cra. 1E No. 18A-10, Santafe de Bogota, Colombia

E-mail: da.bedoya52@uniandes.edu.co, nkelkar@uniandes.edu.co, mnowakos@uniandes.edu.co

ABSTRACT: The proton charge and magnetization density distributions can be related to the well known Sachs electromagnetic form factors $G_{E, M}\left(\boldsymbol{q}^{2}\right)$ through Fourier transforms, only in the Breit frame. The Breit frame however moves with relativistic velocities in the Lab and a Lorentz boost must be applied before extracting the static properties of the proton from the corresponding densities. Apart from this, the Fourier transform relating the densities and form factors is inherently a non-relativistic expression. We show that the relativistic corrections to it can be obtained by extending the standard Breit equation to higher orders in its $1 / c^{2}$ expansion. We find that the inclusion of the above corrections reduces the size of the proton as determined from electron proton scattering data by about $4 \%$.

KEywords: Scattering Amplitudes, Electromagnetic Processes and Properties

ARXIV EPRINT: 1410.4228 


\section{Contents}

1 Introduction 1

2 Theoretical approach 2

2.1 Potentials and densities 2

2.2 Higher order Breit equation 3

2.3 Lorentz boost 5

3 Corrected radii and fourth moments $\quad 6$

$\begin{array}{lll}4 & \text { Summary } & 9\end{array}$

$\begin{array}{ll}\text { A Coefficients in the wave function expansion } & 10\end{array}$

\section{Introduction}

Measurements of the ground state properties of the most basic element of the constituents of stable matter, namely, the proton, have intrigued physicists since the sixties until now. The structure of the proton in fact plays an important role in atomic physics where experiments have reached very high precision. Finite size effects (FSE) due to the proton structure can be theoretically included using different methods, with one of them being the Breit equation [1-9] which is a typical example of how one derives coordinate potentials from Quantum Field Theory [10-15]. Using such a method where one evaluates the elastic electron-proton amplitude expanded in powers of $1 / c^{2}$, corrections to the energy levels of the hydrogen atom due to the finite size of the proton have been evaluated [16, 17]. The FSE are included through the elastic electromagnetic form factors obtained from electron proton scattering cross sections $[18,19]$. The electromagnetic form factors as such are an essential part of the description of the properties of the nucleon as they incorporate the probability for a nucleon to absorb a virtual photon of four momentum squared $q^{2}\left(=\left(q^{0}\right)^{2}-(\boldsymbol{q})^{2}\right)$ and probe its interior. In the non-relativistic limit, the Fourier transforms of the form factors in the Breit frame (defined by $q^{0}=0$ ) describe the charge distribution $\rho_{C}(\mathbf{r})$ and magnetization current distribution $\rho_{M}(\mathbf{r})$ in the nucleon respectively [20-23]. An experimental determination of the form factors (and hence the proton charge density distribution) from electron proton scattering can thus enable one to determine the charge radius of the proton. On the other hand, the unprecedented precision of the experimental results in the hydrogen atom also allows one to probe the static properties of one of the components of the hydrogen atom, namely, the proton. The size of the proton for example, can be extracted from precise measurements of the difference in the energy levels or Lamb shifts in the hydrogen atom. Such an extraction performed on the muonic hydrogen atom led to the 
surprising finding that the extracted value of $r_{p}=0.84184(67) \mathrm{fm}$ was much smaller than the world average CODATA value of $0.8768(69) \mathrm{fm}[24,25]$. This so-called "proton puzzle" was later reinforced $[26,27]$ with the precise value of $r_{p}=0.84087(39)$ fm obtained from muonic hydrogen spectroscopy. Apart from some determinations from standard hydrogen atom spectroscopy, the CODATA value largely depends on the extraction of the radius from electron proton scattering experiments. The shrunk proton gave rise to explanations ranging from the charge density being poorly constrained by data [28] to those involving large extra dimensions and non-identical protons [29].

In an attempt to resolve the discrepancy between the proton radius from muonic hydrogen spectroscopy and electron proton scattering data, we re-examine the connection of the electromagnetic form factors to the nucleon properties. We present a new approach to relate the form factors in momentum space to their coordinate space counter parts (the charge and magnetization densities) through a Fourier transform of the type $\rho_{C}(\mathbf{r})=e \int e^{i \boldsymbol{q} \cdot \mathbf{r}} \rho_{C}\left(\boldsymbol{q}^{2}\right) d^{3} q /(2 \pi)^{3}$. The standard non-relativistic expression is obtained when $\rho_{C}\left(\boldsymbol{q}^{2}\right)=G_{E}\left(\boldsymbol{q}^{2}\right)$, where $G_{E}\left(\boldsymbol{q}^{2}\right)$ is the well known Sachs form factor [30]. The relativistic corrections for $\rho_{C}(\mathbf{r})$ are incorporated by evaluating $\rho_{C}\left(\boldsymbol{q}^{2}\right)$ in the form $\rho_{C}\left(\boldsymbol{q}^{2}\right)=$ $G_{E}\left(\boldsymbol{q}^{2}\right)\left[1+\right.$ terms $\frac{1}{c^{2}}+$ terms $\left.\frac{1}{c^{4}}+\ldots\right]$ using the higher order Breit equation which we derive in this work. Since such a relation is still valid only in the Breit frame (i.e. $q^{0}=0$ ), a Lorentz boost must be applied to $\rho_{C}\left(\boldsymbol{q}^{2}\right)$ before extracting the mean radius from $r_{p}^{2}=\int \rho_{C}(\mathbf{r}) r^{2} d \mathbf{r}$. We find that the inclusion of the two effects (Lorentz boost and use of the higher order Breit equation) brings the radius extracted from electron proton scattering quite close to that determined from the muonic hydrogen Lamb shift [26, 27], thus partly resolving the proton radius puzzle.

\section{Theoretical approach}

The relativistic corrections to the non-relativistic $\rho_{C}(\mathbf{r})$ are obtained by extending the standard Breit equation [16, 17] (which involves an expansion of the amplitude to order $\left.1 / c^{2}\right)[5-7,31,32]$ to higher orders. The proton electric potential $V_{p}(\mathbf{r})$ in this equation is used to find the density $\rho_{C}(\mathbf{r})$ via the Poisson equation, $\nabla^{2} V_{p}=-4 \pi \rho_{C}$. The hyperfine interaction terms in the Breit equation are shown to be related to the magnetization density $\rho_{M}(\mathbf{r})$. In what follows, we shall see that an interesting outcome of the calculation is that the charge form factor $\rho_{C}\left(\boldsymbol{q}^{2}\right)$ appearing in the Fourier transform, depends on the magnetic form factor $G_{M}\left(\boldsymbol{q}^{2}\right)$ and $\rho_{M}\left(\boldsymbol{q}^{2}\right)$ appearing in the Fourier transform of the magnetization density, $\rho_{M}(\mathbf{r})$, depends on $G_{E}\left(\boldsymbol{q}^{2}\right)$.

\subsection{Potentials and densities}

In order to make the approach clear let us begin with the standard Breit equation for the Hamiltonian $H_{B}$ [16] which results from the $1 / c^{2}$ expansion of the elastic electron proton transition matrix element $M_{f i}$. This amplitude can be written as, $M_{f i}=w_{S_{e}^{\prime}}^{\dagger} w_{S_{p}^{\prime}}^{\dagger} \hat{\mathrm{H}}_{B}\left(\mathbf{p}_{e}, \mathbf{p}_{p}\right.$; $\left.\boldsymbol{\sigma}_{e}, \boldsymbol{\sigma}_{p} ; \boldsymbol{q}\right) w_{S_{e}} w_{S_{p}}$, where $w_{S_{e}, S_{p}}$ are two component spinors. In the diagonal case, $S_{e}^{\prime}=S_{e}$, $S_{p}^{\prime}=S_{p}$ and we write $M_{f i}=M_{f i}\left(\mathbf{p}_{e}, \mathbf{p}_{p} ; \boldsymbol{\xi}_{e}, \boldsymbol{\xi}_{p} ; \boldsymbol{q}\right)$, where we used $w_{S}^{\dagger} \boldsymbol{\sigma} w_{S}=\operatorname{Tr}[\rho \boldsymbol{\sigma}]=\boldsymbol{\xi}$ 
with $\rho$ being the spin density matrix. Let us now rearrange terms from the Breit Hamiltonian $H_{B}$ such that, $H_{B}=e V_{p}(\boldsymbol{q})+\mu_{e} \cdot \mathbf{B}(\boldsymbol{q})+\ldots$, where, $\mu_{e}=-\left(e / 2 m_{e}\right) \boldsymbol{\sigma}_{e} \cdot V_{p}(\boldsymbol{q})$ is the potential part remaining after separating all the $\boldsymbol{\sigma}_{i}$ operator dependent and differential operator $\mathbf{p}_{i}$ dependent terms. Here $i$ is either $e$ or $p$. In addition, we choose $V_{p}(\boldsymbol{q})$ not to contain the electron mass as the electric proton potential should not depend on the probe. These restrictions allow $V_{p}(\boldsymbol{q})$ to be interpreted as a proton electric potential in momentum space. For the standard Breit equation at lowest order in $1 / c^{2}$, with form factors, this indeed leads to [17],

$$
V_{p}(\boldsymbol{q})=4 \pi e\left[F_{1}\left(\frac{1}{\boldsymbol{q}^{2}}\right)-F_{2}\left(\frac{1}{4 m_{p}^{2} c^{2}}\right)\right]=4 \pi e\left[\frac{G_{E}\left(\boldsymbol{q}^{2}\right)}{\boldsymbol{q}^{2}}\right],
$$

where $e$ is the positive charge of the proton. The Fourier transform of $V_{p}(\boldsymbol{q})$ is then the electric potential $V_{p}(\mathbf{r})=4 \pi e \int e^{i \boldsymbol{q} \cdot \mathbf{r}}\left(G_{E}\left(\boldsymbol{q}^{2}\right) / \boldsymbol{q}^{2}\right) d^{3} q /(2 \pi)^{3}$. The Laplacian of $V_{p}(\mathbf{r})$, namely, $\nabla^{2} V_{p}(\mathbf{r})=-4 \pi e \int e^{i \boldsymbol{q} \cdot \mathbf{r}} G_{E}\left(\boldsymbol{q}^{2}\right) d^{3} q /(2 \pi)^{3}$ taken together with $\nabla^{2} V_{p}(\mathbf{r})=-4 \pi \rho_{C}(\mathbf{r})$ then brings us to the standard definition of the proton charge density $\rho_{C}(\mathbf{r})=e \int e^{i \boldsymbol{q} \cdot \mathbf{r}} G_{E}\left(\boldsymbol{q}^{2}\right)$ $d^{3} q /(2 \pi)^{3}$. Applying similar restrictions to the magnetic field in the second term in $H_{B}$, i.e., the magnetic field of the proton $B(\boldsymbol{q})$ should not contain any electron mass or operator $\mathbf{p}_{i}$ dependence, the terms which remain (apart from the Coulomb term) are those corresponding to the hyperfine interaction. The hyperfine interaction potential with form factors [33] is given as,

$$
\begin{aligned}
V(\boldsymbol{q})_{\mathrm{hfs}} & =\alpha\left[\frac{\left(\boldsymbol{\sigma}_{e} \cdot \boldsymbol{\sigma}_{p}\right)}{4 m_{e} m_{p} c^{2}}-\frac{\left(\boldsymbol{\sigma}_{e} \cdot \boldsymbol{q}\right) \cdot\left(\boldsymbol{\sigma}_{p} \cdot \boldsymbol{q}\right)}{4 m_{e} m_{p} c^{2} \boldsymbol{q}^{2}}\right] G_{M}\left(\boldsymbol{q}^{2}\right) \\
& =\mu_{e} \cdot \mathbf{B}(\boldsymbol{q}),
\end{aligned}
$$

with $\mu_{e}=-\left(e / 2 m_{e}\right) \sigma_{e}$ as defined earlier. The magnetic field of the proton is thus,

$$
\mathbf{B}(\boldsymbol{q})=e\left[\frac{\boldsymbol{q}\left(\boldsymbol{\sigma}_{p} \cdot \boldsymbol{q}\right)-\boldsymbol{\sigma}_{p} \boldsymbol{q}^{2}}{2 m_{p} c^{2} \boldsymbol{q}^{2}}\right] G_{M}\left(\boldsymbol{q}^{2}\right) .
$$

Taking the Fourier transform of $\mathbf{B}(\boldsymbol{q})$ and using the static Maxwell equation $\nabla \times \mathbf{B}(\mathbf{r})=$ $4 \pi \mathbf{j}(\mathbf{r})$, we can identify

$$
\mathbf{j}(\mathbf{r})=\frac{e}{4 \pi} \int \frac{d^{3} q}{(2 \pi)^{3}} e^{i \boldsymbol{q} \cdot \mathbf{r}} \frac{G_{M}\left(\boldsymbol{q}^{2}\right)(i \boldsymbol{q} \times \boldsymbol{\sigma})}{2 m_{p} c^{2}},
$$

thus implying $\mathbf{j}(\mathbf{r}) \propto \nabla \times \mathbf{M}$ with $\mathbf{M}=\rho_{M}(\mathbf{r}) \boldsymbol{\sigma}_{p}$ which defines the proton magnetization distribution $\rho_{M}(\mathbf{r})=\int e^{i \boldsymbol{q} \cdot \mathbf{r}} G_{M}\left(\boldsymbol{q}^{2}\right) d^{3} q /(2 \pi)^{3}$. In the diagonal case, by the replacement of $\boldsymbol{\sigma}$ by the polarization vector $\boldsymbol{\xi}$, we can conclude that polarized protons will have a magnetic field of the form, $\mathbf{B}(\mathbf{r}) \propto(\boldsymbol{\xi} \cdot \boldsymbol{\nabla}) \boldsymbol{\nabla} \tilde{\rho}_{M}-\boldsymbol{\xi} \rho_{M}$, where, $\tilde{\rho}_{M}=\int e^{i \boldsymbol{q} \cdot \mathbf{r}}\left(G_{M}\left(\boldsymbol{q}^{2}\right) / \boldsymbol{q}^{2}\right) d^{3} q /(2 \pi)^{3}$. All the conclusions drawn above and derived at the lowest order in the relativistic expansion are, of course, valid if we include relativistic corrections.

\subsection{Higher order Breit equation}

The procedure to obtain the Breit potential at higher orders using the electron proton scattering amplitude is exactly the same as that described in $[16,17]$ except that the 
proton and electron wave functions which were written in $[16,17]$ using the non-relativistic approximation with corrections up to order $1 / c^{2}$ are now replaced by those containing relativistic corrections up to order $1 / c^{6}$. This is done by using the Foldy Wouthuysen transformation $[34,35], \Psi_{\mathrm{FW}}=U \Psi_{D}$, where

$$
U=\sqrt{\frac{\left(E+m c^{2}\right)}{2 E}}\left(1+\frac{\beta \boldsymbol{\alpha} \cdot \mathbf{p} c}{E+m c^{2}}\right),
$$

$H_{D} \Psi_{D}=E \Psi_{D}, E=\sqrt{\mathbf{p}^{2} c^{2}+m^{2} c^{4}}, H_{\mathrm{FW}} \Psi_{\mathrm{FW}}=\beta E \Psi_{\mathrm{FW}}$ and $\boldsymbol{\alpha}, \beta$ the usual Dirac matrices. It then follows that $[36,37] \Psi_{\mathrm{FW}}=\left[E(1+\beta) / \sqrt{2 E\left(E+m c^{2}\right)}\right] \Psi_{D}$, where, $\Psi_{\mathrm{FW}}$ contains both the positive and negative energy solutions. The upper and lower components $\Psi_{\mathrm{FW}}^{+}$and $\Psi_{\mathrm{FW}}^{-}$of $\Psi_{\mathrm{FW}}$ can be shown to be related to the Dirac upper and lower components $\phi_{D}$ and $\chi_{D}$ respectively as $[36,37]$

$$
\Psi_{\mathrm{FW}}^{+}=\sqrt{\frac{2 E}{E+m c^{2}}}\left(\begin{array}{c}
\phi_{D} \\
0
\end{array}\right), \quad \Psi_{\mathrm{FW}}^{-}=\sqrt{\frac{2 E}{E+m c^{2}}}\left(\begin{array}{c}
0 \\
\chi_{D}
\end{array}\right) .
$$

The relativistic energy $E$ of the particle includes also its rest energy $m c^{2}$ which must be excluded in arriving at a non-relativistic approximation. We must therefore replace $\Psi$ (FW or D) by $\Psi^{\prime}$ defined as $\Psi=\Psi^{\prime} e^{-i m c^{2} t / \hbar}$. This leads to a relation between the upper and lower components $\phi$ and $\chi$ of $\Psi^{\prime}[31,32]$ which is given by,

$$
\chi=\frac{1}{2 m c}\left[1+\frac{E_{S}}{2 m c^{2}}\right]^{-1} \boldsymbol{\sigma} \cdot \mathbf{p} \phi
$$

where $E_{S}$ is the energy eigenvalue in the Schrödinger equation. Identifying the upper component $\Psi_{\mathrm{FW}}^{\prime+}$ of $\Psi_{\mathrm{FW}}$ with the non-relativistic Schrödinger spinor $w$, we get, $w \sqrt{\left(E+m c^{2}\right) / 2 E}=\phi$. Finally, expanding $E=\left(p^{2} c^{2}+m^{2} c^{4}\right)^{1 / 2}$ and replacing for $\phi$ in terms of $w$ in $\chi$, we obtain the spinor to be used in the calculation of the amplitude $M_{f i}=e^{2}\left(\bar{u}_{e}^{\prime} \Gamma_{e}^{\mu} u_{e}\right) D_{\mu \nu}\left(q^{2}\right)\left(\bar{u}_{p}^{\prime} \Gamma_{p}^{\nu} u_{p}\right)$ as

$$
u_{i}=\sqrt{2 m_{i}}\left(\begin{array}{c}
\left(1-\frac{p_{i}^{2}}{8 m_{i}^{2} c^{2}}+\frac{\lambda_{1} p_{i}^{4}}{m_{i}^{4} c^{4}}+\frac{\lambda_{3} p_{i}^{6}}{m_{i}^{6} c^{6}}\right) w_{i} \\
\left(1-\frac{\lambda_{2} p_{i}^{2}}{m_{i}^{2} c^{2}}+\frac{\lambda_{4} p_{i}^{4}}{m_{i}^{4} c^{4}}\right) \frac{\boldsymbol{\sigma}_{i} \cdot \mathbf{p}_{i}}{2 m_{i} c} w_{i}
\end{array}\right),
$$

with $i=e, p$ and $\lambda_{1}=11 / 128, \lambda_{2}=3 / 8, \lambda_{3}=-69 / 1024$ and $\lambda_{4}=31 / 128$. The above spinor should be contrasted with

$$
u_{i}=\sqrt{2 m_{i}}\left(\begin{array}{c}
\left.1-\frac{p_{i}^{2}}{8 m_{i}^{2} c^{2}}\right) w_{i} \\
\frac{\boldsymbol{\sigma}_{i} \cdot \mathbf{p}_{i}}{2 m_{i} c} w_{i}
\end{array}\right),
$$

used to obtain the standard Breit equation [31,32]. Using eq. (2.8) and the vertices $\Gamma_{p}^{\nu}=F_{1}^{p} \gamma^{\mu}+\sigma^{\mu \nu}\left(q_{\nu} / 2 m_{p} c\right) F_{2}^{p}$ and $\Gamma_{e}^{\mu}=\gamma^{\mu}$, the amplitude $M_{f i}$ and hence the Breit equation with form factors is evaluated just as in $[16,17]$. Note that the energy transfer at the vertices is chosen to be zero, i.e., $q^{2}=\omega^{2} / c^{2}-\boldsymbol{q}^{2}$ is replaced by $q^{2}=-\boldsymbol{q}^{2}$. Formally, this is achieved by going to the Breit frame. This is in keeping with the quasistatic approach 
wherein we are going to relate the proton potential obtained from the Breit equation to the charge density via the Poisson equation. The higher order Breit equation with form factors thus obtained is very lengthy and will be given elsewhere. The present work deals with the parts relevant for obtaining the relativistic corrections to the charge and magnetization densities.

The proton electric potential $\tilde{V}_{p}(\boldsymbol{q})$ with relativistic corrections is obtained from the higher order Breit equation in the same manner as explained before for the standard Breit equation. Dropping all terms involving the spin and momentum operators as well as those containing the electron mass, what remains in the higher order Breit equation is

$$
\begin{aligned}
\tilde{V}_{p}(\boldsymbol{q})=4 \pi e \frac{G_{E}\left(\boldsymbol{q}^{2}\right)}{\boldsymbol{q}^{2}} & \left\{1-\frac{\boldsymbol{q}^{2}}{8 m_{p}^{2} c^{2}}+\frac{3}{128} \frac{\boldsymbol{q}^{4}}{m_{p}^{4} c^{4}}-\frac{13}{1024} \frac{\boldsymbol{q}^{6}}{m_{p}^{6} c^{6}}\right. \\
& \left.+\frac{G_{M}\left(\boldsymbol{q}^{2}\right)}{G_{E}\left(\boldsymbol{q}^{2}\right)} \frac{\boldsymbol{q}^{4}}{16 m_{p}^{4} c^{4}}\left[1-\frac{7}{8} \frac{\boldsymbol{q}^{2}}{m_{p}^{2} c^{2}}+\frac{87}{128} \frac{\boldsymbol{q}^{4}}{m_{p}^{4} c^{4}}\right]\right\} .
\end{aligned}
$$

The above equation can be rewritten as $\tilde{V}_{p}(\boldsymbol{q})=4 \pi e \rho_{C}\left(\boldsymbol{q}^{2}\right) / \boldsymbol{q}^{2}$, such that the proton electric potential, $\tilde{V}_{p}(\mathbf{r})=4 \pi e \int e^{i \boldsymbol{q} \cdot \mathbf{r}}\left(\rho_{C}\left(\boldsymbol{q}^{2}\right) / \boldsymbol{q}^{2}\right) d^{3} q /(2 \pi)^{3}$ and $\nabla^{2} \tilde{V}_{p}(\mathbf{r})=$ $-4 \pi e \int e^{i \boldsymbol{q} \cdot \mathbf{r}} \rho_{C}\left(\boldsymbol{q}^{2}\right) d^{3} q /(2 \pi)^{3}=-4 \pi \rho_{C}(\mathbf{r})$. The magnetic form factor $\rho_{M}\left(\boldsymbol{q}^{2}\right)$ including corrections is obtained by examining the hyperfine interaction terms as mentioned before, however, in the higher order Breit equation. Noting that the terms of order $1 / c^{6}$ and higher are of decreasing importance and due to the alternating sign in (2.10), the first four terms in the curly bracket in (2.10) can be approximated as $\left[1+\left(\boldsymbol{q}^{2} / 4 m_{p}^{2} c^{2}\right)\right]^{-1 / 2}$. The expressions for $\rho_{C, M}\left(\boldsymbol{q}^{2}\right)$ can thus be summarized in an expansion effectively as,

$$
\begin{aligned}
& \rho_{C}\left(\boldsymbol{q}^{2}\right) \simeq G_{E}\left(\boldsymbol{q}^{2}\right)\left(1+\frac{\boldsymbol{q}^{2}}{4 m_{p}^{2} c^{2}}\right)^{-1 / 2}+\frac{G_{M}\left(\boldsymbol{q}^{2}\right) \boldsymbol{q}^{4}}{16 m_{p}^{4} c^{4}}\left(1+\frac{a \boldsymbol{q}^{2}}{4 m_{p}^{2} c^{2}}\right)^{-b} \\
& \rho_{M}\left(\boldsymbol{q}^{2}\right) \simeq G_{M}\left(\boldsymbol{q}^{2}\right)\left(1+\frac{\boldsymbol{q}^{2}}{4 m_{p}^{2} c^{2}}\right)^{-1 / 2}-\frac{G_{E}\left(\boldsymbol{q}^{2}\right) \boldsymbol{q}^{2}}{4 m_{p}^{2} c^{2}}\left(1+\frac{a \boldsymbol{q}^{2}}{4 m_{p}^{2} c^{2}}\right)^{-b},
\end{aligned}
$$

with $a=19 / 7$ and $b=49 / 38$. It is interesting that $\rho_{C}\left(\boldsymbol{q}^{2}\right)$ and $\rho_{M}\left(\boldsymbol{q}^{2}\right)$ depend on both the $G_{E}$ and $G_{M}$ Sachs form factors and have relativistic corrections of a similar form with the same exponents $a$ and $b$. Note also that the exponent $-1 / 2$ in the first terms is approximate (in contrast to the exact $\left[1+\left(\boldsymbol{q}^{2} / 4 m_{p}^{2} c^{2}\right)\right]^{-1 / 2}$ in [38-41]). At order $1 / c^{2}$, the expression for $\rho_{C}\left(\boldsymbol{q}^{2}\right) \simeq G_{E}\left(\boldsymbol{q}^{2}\right)\left(1-\boldsymbol{q}^{2} / 8 m_{p}^{2} c^{2}\right)$ is independent of $G_{M}$ as in [38-42], however, the magnetic form factor at order $1 / c^{2}$ reduces to $\rho_{M}\left(\boldsymbol{q}^{2}\right) \simeq G_{M}\left(\boldsymbol{q}^{2}\right)\left(1-\boldsymbol{q}^{2} / 8 m_{p}^{2} c^{2}\right)-G_{E}\left(\boldsymbol{q}^{2}\right) \boldsymbol{q}^{2} / 4 m_{p}^{2} c^{2}$ and contains apart from the Darwin term $\boldsymbol{q}^{2} / 8 m_{p}^{2}$, a term dependent on $G_{E}$.

\subsection{Lorentz boost}

Since we chose the energy transfer in the evaluation of the electron - proton scattering amplitude, $\omega=0$, the above form factors are similar to those usually given in the so-called Breit frame. An additional important relativistic correction arises due to the Lorentz contraction of the spatial distributions in the Breit frame [43-45]. The latter has been discussed at length in the first reference of [43-45] where the author proposes the use of the Fourier transform of $G_{E, M}^{L}\left(\boldsymbol{q}^{2}\right)=G_{E, M}\left(\boldsymbol{q}^{2}\right)\left[1+\left(\boldsymbol{q}^{2} / 4 m_{p}^{2}\right)\right]^{\lambda_{E, M}}$, rather than the Fourier 
transform of $G_{E, M}\left(\boldsymbol{q}^{2}\right)$ in order to determine the density distributions of the nucleon. With $\lambda_{E, M}$ being model dependent constants, they eventually appear as parameters in the determination of the proton radius and other moments. The author in the first reference in [43-45] fitted the form factor data to obtain $\lambda_{E}=\lambda_{M}=2$ in agreement with some [46] while in contrast with other predictions [47-49] of $\lambda_{E}=0$ and $\lambda_{E}=\lambda_{M}=1$ based on soliton and cluster models.

\section{Corrected radii and fourth moments}

The standard way of defining the $n^{\text {th }}$ moment of the charge and magnetization distribution in literature [50] follows from a consideration of the Fourier transforms of the Sachs form factors in the Breit frame. It makes sense to dwell a little bit on the basics of the definition of the second moment, i.e.,

$$
\left\langle r^{2}\right\rangle=\int r^{2} \rho(\mathbf{r}) d^{3} r
$$

Starting with the Fourier transform of $G\left(\boldsymbol{q}^{2}\right)$, namely, $G\left(\boldsymbol{q}^{2}\right)=\int e^{-i \vec{q} \cdot \vec{r}} \rho(\mathbf{r}) d^{3} r /(2 \pi)^{3}$ we can readily show that

$$
\begin{aligned}
G\left(\boldsymbol{q}^{2}\right) & =\frac{1}{2 \pi^{2}} \int_{0}^{\infty} r^{2} \rho(r) \frac{\sin (|\boldsymbol{q}| r)}{|\boldsymbol{q}| r} d r \\
& =\frac{1}{2 \pi^{2}} \frac{1}{|\boldsymbol{q}|} \int_{0}^{\infty} r \rho(r)\left[|\boldsymbol{q}| r-\frac{|\boldsymbol{q}|^{3} r^{3}}{6}+\ldots\right] \\
& =\frac{1}{2 \pi^{2}}\left[\int_{0}^{\infty} r^{2} \rho(r) d r-\frac{\boldsymbol{q}^{2}}{6} \int_{0}^{\infty} r^{4} \rho(r) d r+\ldots\right]
\end{aligned}
$$

leads to the standard result

$$
-\left.\frac{6}{G(0)} \frac{d G\left(\boldsymbol{q}^{2}\right)}{d \boldsymbol{q}^{2}}\right|_{\boldsymbol{q}^{2}=0}=\int r^{4} \rho(r) d r=\left\langle r^{2}\right\rangle .
$$

Eq. (3.2) is equivalent to writing

$$
G\left(\boldsymbol{q}^{2}\right) / G(0)=1-\frac{1}{6}\left\langle r^{2}\right\rangle \boldsymbol{q}^{2}+\frac{1}{120}\left\langle r^{4}\right\rangle \boldsymbol{q}^{4}-\ldots,
$$

where $\left\langle r^{n}\right\rangle$ is the $n^{\text {th }}$ moment of the electric or magnetic distribution. Neither (3.1) nor (3.2) are relativistic invariants. The form factor $G$ which depends on the four momentum transfer is an invariant and sometimes one finds in the literature the expansion

$$
G\left(q^{2}\right)=1+a q^{2}+\ldots,
$$

with $q^{2}=q_{\mu} q^{\mu}$ being the four-momentum transfer. This is then followed by an expression of the first moment proportional to $d G\left(q^{2}\right) / d q^{2}$ taken at $q^{2}=0$. This in turn might lead to the confusing conclusion that the proton radius is a Lorentz invariant. To resolve the confusion let us first note that we would get the same result by writing $G\left(\omega=0, \boldsymbol{q}^{2}\right)=1-a \boldsymbol{q}^{2}+\ldots$ and taking the derivative with respect to $\boldsymbol{q}^{2}$ evaluated at $\boldsymbol{q}^{2}=0$ which agrees with (3.3) and, of course, (3.1). Therefore, we would face a paradox here: by using (3.5) and its 
derivative with respect to the four momentum squared $q^{2}$, it seems like we have found a Lorentz invariant quantity and this is equivalent to a Lorentz non-invariant result (up to the minus sign which is absorbed into the definition). The resolution of the paradox lies in the meaning of the condition, $q^{2}=0$. With $q^{2}=\omega^{2}-\boldsymbol{q}^{2}$, it either means that $\omega^{2}=\boldsymbol{q}^{2} \neq 0$ (in which case we have a real photon) or $\omega=|\boldsymbol{q}|=0$. It is impossible to exchange a real photon in the t-channel exchange diagram in elastic electron-proton scattering and hence we have to drop the first possibility. The second choice is, however, equivalent to first choosing the frame ( $\omega=0$ implies that we have chosen the Breit frame again) and then $\boldsymbol{q}^{2}=0$ is necessary to extract the Taylor coefficient (the radius). In short, even if $d G\left(q^{2}\right) / d q^{2}$ is invariant, the condition $q^{2}=0$ makes the radius defined using $d G\left(q^{2}\right) / d q^{2}$ at $q^{2}=0$, a Lorentz non-invariant quantity (as the condition forces one to choose $\omega=0$ ).

If $\rho_{C}(\mathbf{r})$ and $\rho_{M}(\mathbf{r})$ (defined by $\rho_{C}(\mathbf{r})=e \int e^{i \boldsymbol{q} \cdot \mathbf{r}} \rho_{C}\left(\boldsymbol{q}^{2}\right) d^{3} q /(2 \pi)^{3}$ and $\rho_{M}(\mathbf{r})=$ $\left.\int e^{i \boldsymbol{q} \cdot \mathbf{r}} \rho_{M}\left(\boldsymbol{q}^{2}\right) d^{3} q /(2 \pi)^{3}\right)$, receive relativistic corrections as given in (2.11), so will the corresponding radii. Hence, the proton moments including the relativistic corrections and the Lorentz boost are defined here as:

$$
\left\langle\tilde{r}_{E}^{2}\right\rangle^{L}=-\left.\frac{6}{\rho_{C}^{L}(0)} \frac{d \rho_{C}^{L}}{d \boldsymbol{q}^{2}}\right|_{\boldsymbol{q}^{2}=0}
$$

and

$$
\left\langle\tilde{r}_{E}^{4}\right\rangle^{L}=\left.\frac{60}{\rho_{C}^{L}(0)} \frac{d^{2} \rho_{C}^{L}}{d\left(\boldsymbol{q}^{2}\right)^{2}}\right|_{\boldsymbol{q}^{2}=0}
$$

(with $\left.\rho_{C}^{L}\left(\boldsymbol{q}^{2}\right)=\rho_{C}\left(\boldsymbol{q}^{2}\right)\left[1+\left(\boldsymbol{q}^{2} / 4 m_{p}^{2} c^{2}\right)\right]^{\lambda_{E}}\right)$. Replacing from (2.11) for $\rho_{C}\left(\mathbf{q}^{2}\right)$ leads to

$$
\begin{aligned}
\left\langle\tilde{r}_{E}^{2}\right\rangle^{L}= & \left\langle r_{E}^{2}\right\rangle+\frac{3}{4 m_{p}^{2} c^{2}}\left(1-2 \lambda_{E}\right), \\
\left\langle\tilde{r}_{E}^{4}\right\rangle^{L}= & \left\langle r_{E}^{4}\right\rangle-\frac{5}{m_{p}^{2} c^{2}}\left\langle r_{E}^{2}\right\rangle\left(\lambda_{E}-\frac{1}{2}\right) \\
& +\frac{15}{4 m_{p}^{4} c^{4}}\left(\lambda_{E}^{2}-2 \lambda_{E}+2 \mu_{p}\right)+\frac{45}{16 m_{p}^{4} c^{4}} .
\end{aligned}
$$

The magnetic radius with relativistic and Lorentz boost corrections is given by,

$$
\left\langle\tilde{r}_{M}^{2}\right\rangle^{L}=\left\langle r_{M}^{2}\right\rangle+\frac{3}{4 m_{p}^{2} c^{2}}\left[1+\frac{2}{\mu_{p}}-2 \lambda_{M}\right] .
$$

The relativistic corrections alone (giving $\tilde{r}_{p}$ and $\tilde{r}^{4}$ in table 1) arising from (2.11) can be found by setting $\lambda_{E, M}=0$.

The effect of the Lorentz boost in general is to reduce the radius and the fourth moment of the proton charge as compared to that obtained from $G_{E}\left(\boldsymbol{q}^{2}\right)$ in the Breit frame. The relativistic corrections introduced with the use of $\rho_{C}\left(\boldsymbol{q}^{2}\right)$ obtained from the higher order Breit potential, in general, increase the radius of the proton. However, a fortuitous combination of the two effects, brings the proton radius closer to the value obtained from precise Lamb shift measurements [26, 27]. Indeed, if we apply the Lorentz boost with $\lambda=1$ to the central value of the radius $r_{p}=0.879 \mathrm{fm}$ deduced recently by 


\begin{tabular}{|l|c|c|c|c|}
\hline & $\begin{array}{c}r_{p} \\
\left(r^{4}\right)\end{array}$ & $\begin{array}{c}\tilde{r}_{p} \\
\left(\tilde{r}^{4}\right)\end{array}$ & $\begin{array}{c}\tilde{r}_{p}^{L} \\
\left(\tilde{r}^{4}\right)^{L} \\
\lambda_{E}=1\end{array}$ & $\begin{array}{c}\tilde{r}_{p}^{L} \\
\left(\tilde{r}^{4}\right)^{L} \\
\lambda_{E}=2\end{array}$ \\
\hline Dipole $[18,19]$ & 0.811 & 0.831 & 0.790 & 0.747 \\
up to $1 / c^{4}$ & $(1.083)$ & $(1.202)$ & $(1.049)$ & $(0.911)$ \\
up to $1 / c^{2}$ & $(1.083)$ & $(1.156)$ & $(1.010)$ & $(0.864)$ \\
\hline [52] Fit I & 0.884 & 0.903 & 0.865 & 0.826 \\
up to $1 / c^{4}$ & $(1.788)$ & $(1.920)$ & $(1.740)$ & $(1.574)$ \\
up to $1 / c^{2}$ & $(1.788)$ & $(1.875)$ & $(1.702)$ & $(1.529)$ \\
\hline [52] Fit II & 0.866 & 0.885 & 0.847 & 0.807 \\
up to $1 / c^{4}$ & $(1.623)$ & $(1.752)$ & $(1.579)$ & $(1.420)$ \\
up to $1 / c^{2}$ & $(1.623)$ & $(1.706)$ & $(1.540)$ & $(1.374)$ \\
\hline [53] & 0.858 & 0.877 & 0.839 & 0.798 \\
up to $1 / c^{4}$ & $(1.488)$ & $(1.616)$ & $(1.446)$ & $(1.290)$ \\
up to $1 / c^{2}$ & $(1.488)$ & $(1.570)$ & $(1.407)$ & $(1.244)$ \\
\hline
\end{tabular}

Table 1. Corrections to the proton charge radius $r_{p}=\left\langle r_{E}^{2}\right\rangle^{1 / 2}$ in $\mathrm{fm}$. The fourth moments $r^{4}=\left\langle r_{E}^{4}\right\rangle$ of the proton charge distribution with corrections (up to order $1 / c^{2}$ and $1 / c^{4}$ ) are given in the brackets $\left(\right.$ in $\left.\mathrm{fm}^{4}\right)$. The first column gives the usual uncorrected values obtained from $e p$ scattering, the second column shows the increase in values due to relativistic corrections and finally the last two columns display the effects of relativistic corrections and the Lorentz boost taken together.

Bernauer et al. [50], we obtain $r_{p}^{L}=0.8404 \mathrm{fm}$ which is once again close to the muonic hydrogen spectroscopy result $[26,27]$. This is demonstrated in figure 1 . The reason for applying only the Lorentz boost and not the entire relativistic corrections is the following: Bernauer et al. include in their analysis, the "Feshbach correction" which as stated above eq. (20) in [50] is in agreement with the Coulomb correction of Rosenfelder [38] at $\boldsymbol{q}^{2}=0$. This correction of Rosenfelder is similar to the relativistic corrections of the present paper upto order $1 / c^{2}$ (compare eq. (7) of [38] with the first term in eq. (2.11) of the present work). It would lead to a double counting if we would apply the relativistic correction of our work to the radius of Bernauer et al. and hence we apply only the Lorentz boost. Though we do not show explicitly, similar corrections would also shift the other radii in figure 1, extracted from $e p$ scattering, to lower values. The proton magnetic radius, $r_{M}=0.87 \mathrm{fm}$ [54], with relativistic and Lorentz boost corrections changes to $r_{M}=0.865 \mathrm{fm}$. We must emphasize however that the proton is characterized fully by all its moments and not just the radius. The corrections in eqs. (2.11) introduce a significant change in $\left\langle r^{4}\right\rangle$ too.

Having mentioned the numerical values obtained after applying relativistic corrections and the Lorentz boost, a word of caution is however in order here. Electron proton scattering measures transition matrix elements between states of a composite system that have different momenta and the transition densities between such states are different from the static densities in the rest frame. As a result, there arise uncertainties in the way the Lorentz boost is calculated (see the third reference in [43-45] for a detailed discussion). For example, in the first calculation done by Licht and Pagnamenta [47-49], the authors had to 


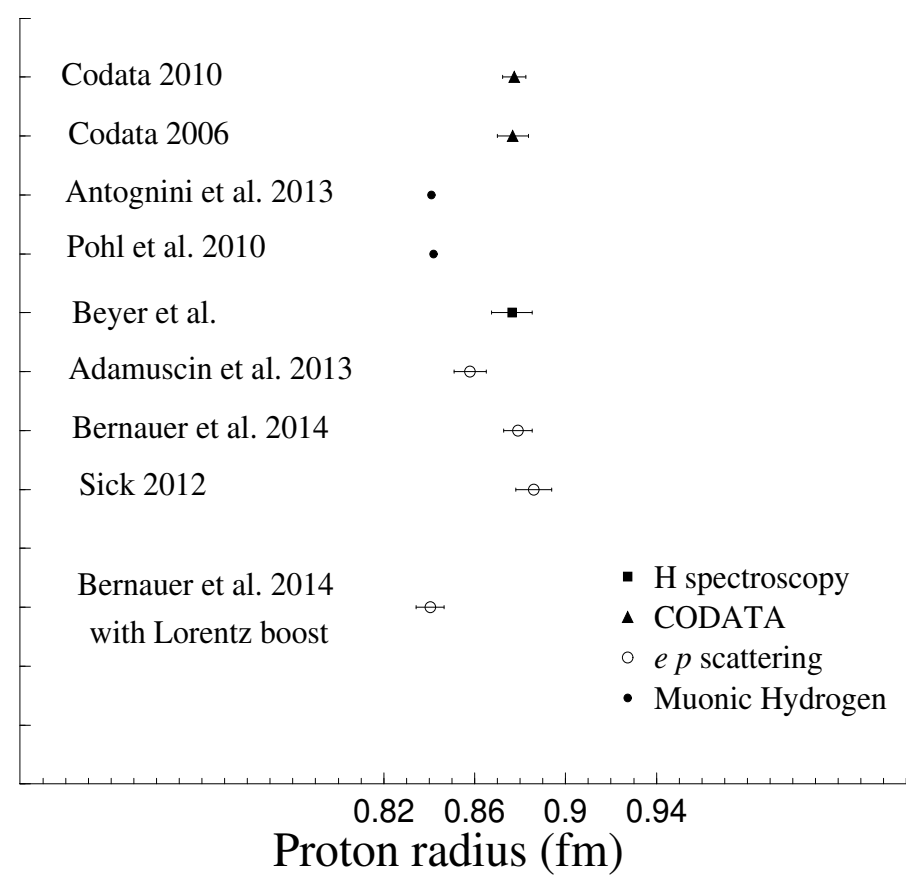

Figure 1. Comparison of the proton radius values extracted from the muonic hydrogen Lamb shift $[26,27]$, CODATA values $[24,25]$ and some recent analyses $[28,50]$ of $e p$ scattering data. The hydrogen spectroscopy average is from [51].

apply a sort of impulse approximation assuming that the transition from the initial to the final cluster happens instantaneously. Further problems were noted in the second reference in [47-49] where the authors calculated the form factors within a chiral soliton model. We also refer the reader to a more recent calculation [55] where some of the important boost effects associated with the use of the Breit frame were studied within a toy model using a harmonic oscillator basis. Taking all this into account, we come to the conclusion that even if the Lorentz boost is essential, the exact method of applying it is not well known. Hence, instead of emphasizing the exact values, we conlcude this section by mentioning that the relativistic corrections and the Lorentz boost taken together cause a reduction of about $4 \%$ in the radius of the proton calculated from electron proton scattering.

In passing we mention that the proton structure corrections as such are also dependent on the theoretical formalism used to calculate them. We refer the reader to ref. [16] for a detailed discussion of the proton structure corrections using different formalisms.

\section{Summary}

The relations between charge/magnetization densities and the electromagnetic form factors are necessarily of a non-relativistic nature. In other words, relativistic corrections can be computed and the standard relation between the Sachs form factors $\left(G_{E}\left(\boldsymbol{q}^{2}\right)\right.$ and $\left.G_{M}\left(\boldsymbol{q}^{2}\right)\right)$ and the densities is valid only at the lowest order of the non-relativistic expansion. To compute the relativistic corrections in a consistent way we employed the higher order Breit 
equation in which, for instance, terms independent of the probe, spin and momentum operators should correspond to the proton electric potential in momentum space. Using the Poisson equation, this potential gives us the relativistically modified charge density. A similar procedure can be found for the magnetization density. Both results are valid in the Breit frame. Hence using a Lorentz transformation suggested in the literature, we can bring them to the rest frame of the proton and calculate the modified moments of the proton charge and magnetization densities. An interesting outcome of the manipulations, i.e., including relativistic corrections and the Lorentz transformation is that the proton radius from $e p$ scattering experiments comes closer to the result obtained from muonic hydrogen spectroscopy.

\section{A Coefficients in the wave function expansion}

A free spin $1 / 2$ particle is described by a four component wave function satisfying the Dirac equation. It is however, often desirable to convert this equation to a two component equation of the Pauli type. Methods attempting to do this however encounter difficulties if one wishes to go beyond the lowest order in the $v / c$ expansion. A method proposed by Foldy and Wouthuysen $[34,35]$ however overcomes these difficulties. Their treatment involves a unitary transformation which block diagonalizes the Dirac Hamiltonian and eventually splits the Dirac equation into two uncoupled equations of the Pauli type, describing particles in positive- and negative-energy states, respectively. Since the procedure to carry out the Foldy-Wouthuysen transformation is given below eq. (2.5) in the main text, here we only write the intermediate steps for obtaining the coefficients in eq. (2.8).

We start with $w \sqrt{\left(E+m c^{2}\right) / 2 E}=\phi$ as given below eq. (2.7) and expanding $E=$ $\left(p^{2} c^{2}+m^{2} c^{4}\right)^{1 / 2}$, we obtain,

$$
\phi=\left[1-\frac{p^{2}}{8 m^{2} c^{2}}+\frac{11}{128} \frac{p^{4}}{m^{4} c^{4}}-\frac{69}{1024} \frac{p^{6}}{m^{6} c^{6}} \ldots\right] w .
$$

This is the upper component given in eq. (2.8). Replacing the above $\phi$ in eq. (2.7) namely,

$$
\begin{aligned}
\chi & =\frac{1}{2 m c}\left[1+\frac{E_{S}}{2 m c^{2}}\right]^{-1} \boldsymbol{\sigma} \cdot \mathbf{p} \phi, \\
& =\frac{\boldsymbol{\sigma} \cdot \mathbf{p}}{2 m c}\left(1-\frac{E_{S}}{2 m c^{2}}+\frac{E_{S}^{2}}{4 m^{2} c^{4}}-\frac{E_{S}^{3}}{8 m^{3} c^{6}}+\ldots\right) \phi,
\end{aligned}
$$

using the fact that $E_{S} w=\hat{H} w$ where,

$$
\hat{H}=\frac{p^{2}}{2 m}-\frac{p^{4}}{8 m^{3} c^{2}}
$$

and replacing accordingly for every $E_{S} w$ by $\left[\frac{p^{2}}{2 m}-\frac{p^{4}}{8 m^{3} c^{2}}\right] w$, we get

$$
\begin{aligned}
\chi & =\frac{\boldsymbol{\sigma} \cdot \mathbf{p}}{2 m c}\left[1-\frac{p^{2}}{4 m^{2} c^{2}}+\frac{p^{4}}{8 m^{4} c^{4}}-\ldots\right]\left[1-\frac{p^{2}}{8 m^{2} c^{2}}+\frac{11}{128} \frac{p^{4}}{m^{4} c^{4}} \ldots\right] w \\
& =\left[1-\frac{3}{8} \frac{p^{2}}{m^{2} c^{2}}+\frac{31}{128} \frac{p^{4}}{m^{4} c^{4}} \ldots\right] \frac{\boldsymbol{\sigma} \cdot \mathbf{p}}{2 m c} w .
\end{aligned}
$$

This is the lower component in eq. (2.8). 
We investigated another method $[31,32,56]$ to obtain such an expansion and it was gratifying to find the same coefficients as above. If we begin again with eq. (2.7) as the starting point and perform an expansion for $\chi$ after noting that $E_{S}$ is the total energy with rest energy subtracted, $\chi$ can be rewritten as,

$$
\chi=c \boldsymbol{\sigma} \cdot \mathbf{p}\left[\frac{1}{2 m c^{2}}-\frac{p^{2}}{8 m^{3} c^{4}}+\frac{p^{4}}{16 m^{5} c^{6}}-\ldots\right] \phi .
$$

Now noting that the density $\rho=\Psi^{*} \Psi=|\chi|^{2}+|\phi|^{2}$, we obtain for the density,

$$
\rho=|\phi|^{2}+c^{2}\left[\left(\mathbf{p}^{*} A \phi^{\dagger}\right) \cdot \boldsymbol{\sigma}\right][\boldsymbol{\sigma} \cdot(\mathbf{p} A \phi)],
$$

where $A$ is basically the operator in square brackets in (A.3). This $\rho$ obviously differs from the Schrödinger expression. In order to find the wave equation corresponding to the Schrödinger equation, we must replace $\phi$ by another function $\phi_{\text {Sch }}$, for which the time independent integral would be of the form $\int\left|\phi_{\mathrm{Sch}}\right|^{2} d^{3} x$ as it should be for the Schrödinger equation. Hence, to obtain the required transformation, we write the condition

$$
\int\left|\phi_{\mathrm{Sch}}\right|^{2} d^{3} x=\int\left\{|\phi|^{2}+c^{2}\left[\left(\mathbf{p}^{*} A \phi^{\dagger}\right) \cdot \boldsymbol{\sigma}\right][\boldsymbol{\sigma} \cdot(\mathbf{p} A \phi)]\right\} d^{3} x .
$$

Integrating the second term by parts and after some lengthy but straightforward algebra, we find that the following expression for $\phi$, satisfies the relation in (A.5).

$$
\phi=\left[1-\frac{p^{2}}{8 m^{2} c^{2}}+\frac{11}{128} \frac{p^{4}}{m^{4} c^{4}}-\frac{69}{1024} \frac{p^{6}}{m^{6} c^{6}} \ldots\right] \phi_{\text {Sch }} .
$$

This is however the same as eq. (A.1) for the upper component. Replacing this $\phi$ in (A.3), obviously leads to the same lower component as in (A.2).

Open Access. This article is distributed under the terms of the Creative Commons Attribution License (CC-BY 4.0), which permits any use, distribution and reproduction in any medium, provided the original author(s) and source are credited.

\section{References}

[1] G. Breit, The effect of retardation on the interaction of two electrons, Phys. Rev. 34 (1929) 553 [inSPIRE].

[2] G. Breit, The fine structure of HE as a test of the spin interactions of two electrons, Phys. Rev. 36 (1930) 383 [inSPIRE].

[3] G. Breit, Dirac's equation and the spin-spin interactions of two electrons, Phys. Rev. 39 (1932) 616.

[4] J.R. Oppenheimer, Note on the theory of the interaction of field and matter, Phys. Rev. 35 (1930) 461 [inSPIRE].

[5] M. De Sanctis and P. Quintero, Charmonium spectrum with a generalized Fermi-Breit equation, Eur. Phys. J. A 46 (2010) 213 [InSPIRE]. 
[6] M. De Sanctis, A generalization of the Fermi-Breit equation to non-Coulombic spatial interactions, Eur. Phys. J. A 41 (2009) 169 [inSPIRE].

[7] M. De Sanctis, A relativistic wave equation with a local kinetic operator and an energy-dependent effective interaction for the study of hadronic systems, Centr. Eur. J. Phys. 12 (2014) 221.

[8] D.A. Kulikov and R.S. Tutik, A new two-body relativistic potential model for pionic hydrogen, Mod. Phys. Lett. A 25 (2010) 447 [arXiv:0906.5066] [INSPIRE].

[9] D.A. Kulikov and R.S. Tutik, A new approach to the relativistic treatment of the fermion-boson system, based on the extension of the $\mathrm{SL}(2, C)$ group, Mod. Phys. Lett. A 23 (2008) 1829 [arXiv:0711.4511] [INSPIRE].

[10] V.M. Mostepanenko and I.Y. Sokolov, The restrictions on long-range forces following from Casimir effect, Sov. J. Nucl. Phys. 46 (1987) 685.

[11] J.A. Grifols and S. Tortosa, Residual long range pseudoscalar forces between unpolarized macroscopic bodies, Phys. Lett. B 328 (1994) 98 [hep-ph/9404249] [INSPIRE].

[12] F. Ferrer and J.A. Grifols, Long range forces from pseudoscalar exchange, Phys. Rev. D 58 (1998) 096006 [hep-ph/9805477] [INSPIRE].

[13] F. Ferrer and M. Nowakowski, Higgs and Goldstone bosons mediated long range forces, Phys. Rev. D 59 (1999) 075009 [hep-ph/9810550] [INSPIRE].

[14] J.A. Grifols, Higgsonium, Phys. Lett. B 264 (1991) 149 [InSPIRE].

[15] F. Ferrer, J.A. Grifols and M. Nowakowski, Long range neutrino forces in the cosmic relic neutrino background, Phys. Rev. D 61 (2000) 057304 [hep-ph/9906463] [INSPIRE].

[16] F. Garcia Daza, N.G. Kelkar and M. Nowakowski, Breit equation with form factors in the hydrogen atom, J. Phys. G 39 (2012) 035103 [arXiv: 1008.4384] [INSPIRE].

[17] N.G. Kelkar, F.G. Daza and M. Nowakowski, Determining the size of the proton, Nucl. Phys. B 864 (2012) 382 [arXiv:1203.0581] [INSPIRE].

[18] P.E. Bosted et al., Measurements of the electric and magnetic form-factors of the proton from $Q^{2}=1.75$ to $8.83 \mathrm{GeV} / \mathrm{c}^{2}$, Phys. Rev. Lett. 68 (1992) 3841 [INSPIRE].

[19] P.E. Bosted, An empirical fit to the nucleon electromagnetic form-factors, Phys. Rev. C 51 (1995) 409 [inSPIRE].

[20] C.F. Perdrisat, V. Punjabi and M. Vanderhaeghen, Nucleon electromagnetic form factors, Prog. Part. Nucl. Phys. 59 (2007) 694 [hep-ph/0612014] [InSPIRE].

[21] H. Gao, Hadron physics at low energies, Eur. Phys. J. Spec. Top. 198 (2011) 3 [InSPIRE].

[22] H. Gao, Nucleon electromagnetic form factors, Int. J. Mod. Phys. A 20 (2005) 1595 [INSPIRE].

[23] J. Arrington, Implications of the discrepancy between proton form-factor measurements, Phys. Rev. C 69 (2004) 022201 [nucl-ex/0309011] [INSPIRE].

[24] P.J. Mohr, B.N. Taylor and D.B. Newell, CODATA recommended values of the fundamental physical constants: 2006, Rev. Mod. Phys. 80 (2008) 633 [arXiv:0801.0028] [INSPIRE].

[25] P.J. Mohr, B.N. Taylor and D.B. Newell, CODATA recommended values of the fundamental physical constants: 2010, Rev. Mod. Phys. 84 (2012) 1527 [arXiv:1203.5425] [InSPIRE]. 
[26] A. Antognini et al., Proton structure from the measurement of 2S-2P transition frequencies of muonic hydrogen, Science 339 (2013) 417 [INSPIRE].

[27] R. Pohl et al., The size of the proton, Nature 466 (2010) 213 [INSPIRE].

[28] I. Sick, Problems with proton radii, Prog. Part. Nucl. Phys. 67 (2012) 473 [InSPIRE].

[29] T. Mart and A. Sulaksono, Nonidentical protons, Phys. Rev. C 87 (2013) 025807 [arXiv:1302.6012] [INSPIRE].

[30] F.J. Ernst, R.G. Sachs and K.C. Wali, Electromagnetic form factors of the nucleon, Phys. Rev. 119 (1960) 1105 [INSPIRE].

[31] V.B. Berestetskii, E.M. Lifshitz and L.P. Pitaevskii, Quantum electrodynamics, Landau-Lifshitz Course on Theoretical Physics, Volume 4, 2nd edition, Butterworth-Heinemann, Oxford U.K. (2007).

[32] H.A. Bethe and E.E. Salpeter, Quantum mechanics of one- and two-electron atoms, Dover, New York U.S.A. (2008).

[33] N.G. Kelkar, M. Nowakowski and D. Bedoya Fierro, Opportunities and problems in determining proton and light nuclear radii, Pramana 83 (2014) 761 [INSPIRE].

[34] L.L. Foldy and S.A. Wouthuysen, On the Dirac theory of spin 1/2 particle and its nonrelativistic limit, Phys. Rev. 78 (1950) 29 [INSPIRE].

[35] E. Eriksen, Foldy-Wouthuysen transformation. Exact solution with generalization to the two-particle problem, Phys. Rev. 111 (1958) 1011 [INSPIRE].

[36] A.J. Silenko, Connection between wave functions in the Dirac and Foldy-Wouthuysen representations, Phys. Part. Nucl. Lett. 5 (2008) 501 [math-ph/0612045] [INSPIRE].

[37] V.P. Neznamov and A.J. Silenko, Foldy-Wouthyusen wave functions and conditions of transformation between Dirac and Foldy-Wouthuysen representations,

J. Math. Phys. 50 (2009) 122302 [arXiv:0906. 2069] [INSPIRE].

[38] R. Rosenfelder, Coulomb corrections to elastic electron proton scattering and the proton charge radius, Phys. Lett. B 479 (2000) 381 [nucl-th/9912031] [INSPIRE].

[39] L.L. Foldy, K.W. Ford and D.R. Yennie, Effect of recoil on the elastic scattering of high-energy electrons by zero-spin nuclei, Phys. Rev. 113 (1959) 1147 [INSPIRE].

[40] J.L. Friar and J.W. Negele, Theoretical and experimental determination of nuclear charge distributions, Adv. Nucl. Phys. 8 (1975) 219 [inSPIRE].

[41] J.L. Friar, J. Martorell and D.W.L. Sprung, Nuclear sizes and the isotope shift, Phys. Rev. A 56 (1997) 4579 [nucl-th/9707016] [INSPIRE].

[42] D.R. Yennie, M.M. Lévy and D.G. Ravenhall, Electromagnetic structure of nucleons, Rev. Mod. Phys. 29 (1957) 144.

[43] J.J. Kelly, Nucleon charge and magnetization densities from Sachs form-factors, Phys. Rev. C 66 (2002) 065203 [hep-ph/0204239] [INSPIRE].

[44] D. Robson, Solution to the proton radius puzzle, Int. J. Mod. Phys. E 23 (2014) 1450090 [arXiv: 1305.4552] [INSPIRE].

[45] M.M. Giannini and E. Santopinto, On the proton radius problem, arXiv:1311.0319 [INSPIRE]. 
[46] A.N. Mitra and I. Kumari, Relativistic form-factors for clusters with nonrelativistic wave functions, Phys. Rev. D 15 (1977) 261 [INSPIRE].

[47] A.L. Licht and A. Pagnamenta, Wave functions and form factors for relativistic composite particles. II, Phys. Rev. D 2 (1970) 1156 [InSPIRE].

[48] G. Holzwarth, Electromagnetic nucleon form-factors and their spectral functions in soliton models, Z. Phys. A 356 (1996) 339 [hep-ph/9606336] [INSPIRE].

[49] G. Holzwarth, Electromagnetic form factors of the nucleon in chiral soliton models, hep-ph/0511194 [INSPIRE].

[50] A1 collaboration, J.C. Bernauer et al., Electric and magnetic form factors of the proton, Phys. Rev. C 90 (2014) 015206 [arXiv:1307.6227] [InSPIRE].

[51] A. Beyer et al., Precision spectroscopy of atomic hydrogen, J. Phys. Conf. Ser. 467 (2013) 012003 [inSPIRE].

[52] W.M. Alberico, S.M. Bilenky, C. Giunti and K.M. Graczyk, Electromagnetic form factors of the nucleon: new fit and analysis of uncertainties, Phys. Rev. C 79 (2009) 065204 [arXiv:0812.3539] [INSPIRE].

[53] J. Friedrich and T. Walcher, A coherent interpretation of the form factors of the nucleon in terms of a pion cloud and constituent quarks, Eur. Phys. J. A 17 (2003) 607 [hep-ph/0303054] [INSPIRE].

[54] Z. Epstein, G. Paz and J. Roy, Model independent extraction of the proton magnetic radius from electron scattering, Phys. Rev. D 90 (2014) 074027 [arXiv: 1407.5683] [INSPIRE].

[55] T.W. Donnelly, D.K. Hasell and R.G. Milner, Interpretations of elastic electron scattering, arXiv: 1505.04723 [INSPIRE].

[56] V.B. Berestetskii and L.D. Landau, On the exchange effects between electron and positron, Zh. Eksp. Teor. Fiz. 19 (1949) 673. 